\title{
“EFFECT OF CROSSLINKING AGENT ON DEVELOPMENT OF GASTRORETENTIVE MUCOADHESIVE MICROSPHERES OF RISEDRONATE SODIUM"
}

\author{
SHWETA GEDAM*, PRITEE JADHAV, SWATI TALELE, ANIL JADHAV \\ Sandip Institute of Pharmaceutical Sciences, Mahiravani, Nashik 422213, M. S., India \\ Email: shwetashahare16@gmail.com
}

Received: 19 Mar 2018, Revised and Accepted: 06 Jun 2018

\begin{abstract}
Objective: The present investigation was undertaken to develop and evaluate a gastroretentive mucoadhesive microspheres of anti-osteoporosis drug risedronate sodium to enhance the residence time and drug release by studying the effect of the crosslinking agent to obtain the best
\end{abstract} formulation with reduced particle size and good in vitro mucoadhesion strength.

Methods: Selected drug risedronate sodium is a potent pyridinyl bisphosphonate used for the treatment of osteoporosis, and other bone disorders. Microspheres using sodium alginate as a polymer and calcium chloride solution as a cross-linker were prepared successfully by the emulsification crosslinking method. The $2^{3}$ factorial design was used to study the effects of various variables like a drug: polymer ratio, crosslinking agent concentration and crosslinking time on the particle size and in vitro mucoadhesion strength. All these formulations were evaluated for entrapment efficiency, percentage yield and cumulative drug release. F1 batch was selected as best formulation and evaluated for scanning electron microscopy, fourier transforms infrared spectroscopy, differential scanning calorimetry, stability study.

Results: Design batches were evaluated for percent yield (61.29-89.33\%), \% entrapment efficiency (42.25 $\pm 0.620-62.58 \pm 0.330)$, mucoadhesion strength $(68.15 \pm 0.37-82.24 \pm 0.72 \%)$ and drug release at $12 \mathrm{~h}(67-84 \%)$. Among the microspheres formulation, an F1 batch of (0.5:1) drug: polymer concentration and at $4 \%$ concentration of calcium chloride as a crosslinker was considered best formulation with reduced particle size $32.85 \pm 0.774 \mu \mathrm{m}, \%$ intro mucoadhesion. 82.24 \pm 0.72 . In vitro mucoadhesion strength was increased with the increasing crosslinking time from 5 min to $10 \mathrm{~min}$. The fourier transform infrared spectroscopy (FTIR) and differential scanning calorimetry (DSC) study showed no interaction between drug and polymer. X-ray diffraction (XRD) spectrum of microspheres indicates that drug particles are dispersed at the molecular level in the polymer matrices so no indication of the crystalline nature of the drug nature. Scanning electron microscopic (SEM) study showed that microspheres were spherical in shape with a smooth surface. F1 batch shows percentage cumulative drug release $84.07 \%$. In vitro dissolution studies indicates that percent cumulative drug release from microspheres follows zero order kinetics plot which indicates controlled-release drugdelivery for $12 \mathrm{~h}$ which leads to control of plasma concentration.

Conclusion: The results show that the formulation that contains $(0.5: 1)$ drug: polymer ratio, calcium chloride in $4 \%$ concentration and crosslinking time $10 \mathrm{~min}$ is the best one and can be utilized to formulate risedronate sodium mucoadhesive microspheres to enhance gastric residence time, improved patient compliance and reduction in the frequency of drug administration.

Keywords: Risedronate sodium, Gastro retentive drug delivery system (GRDDS), Emulsification crosslinking method, \% in vitro mucoadhesion

(C) 2018 The Authors. Published by Innovare Academic Sciences Pvt Ltd. This is an open access article under the CC BY license (http://creativecommons.org/licenses/by/4.0/) DOI: http://dx.doi.org/10.22159/ijap.2018v10i4.26071

\section{INTRODUCTION}

Oral formulations have earned a significant place among the various dosage forms developed for human administration. In most of the cases, the conventional oral delivery systems show limited bioavailability because of fast gastric-emptying time among many other reasons involved $[1,2]$. However, the recent technological development has resulted in many novel pharmaceutical products, mainly the controlled release drug delivery systems to overcome this problem. Gastro-retentive drug delivery system (GRDDS) is one such example where the attribute like gastric retention time coupled with the drug release for an extended time has significantly improved patient compliance. Some inherent limitations of the conventional oral drug delivery systems have ignited the interest to this new delivery system. Fast gastric emptying is associated with conventional oral medications leads to a bioavailability issue for many drug molecules [3].

Micro carrier's technology offers a novel approach for drug delivery by coupling the drug to microcarrier particles which modify the release and absorption characteristics of the drug. Microspheres possess important features, among the particulate drug delivery systems by virtue of their small size and efficient carrier characteristics; however, the success of the microspheres delivery system is limited due to their short residence time at the site of absorption. It would, therefore, be advantageous to have the means for providing an intimate contact of the microspheres delivery system with absorbing biological membranes. This can be accomplished by coupling mucoadhesion characteristics to microspheres by using mucoadhesive polymer and developing mucoadhesive microspheres [4]. Mucoadhesive microspheres as modified release formulations maintain the blood levels of the active ingredients for a prolonged period of time and reduces the frequency of drug administration and improve the patient compliance $[5,6]$.

Risedronate is a potent pyridinyl bisphosphonate used for the treatment of osteoporosis, Paget's disease and other bone disorders. Risedronate has a high affinity for hydroxyapatite crystals in bone and is a potent antiresorptive agent. At the cellular level, risedronate inhibits osteoclasts. The osteoclasts adhere normally to the bone surface but show evidence of reduced active resorption. The main reason for lack of therapeutic effectiveness is low oral bioavailability $(0.63 \%)$. The low oral bioavailability of the drug can be attributed to its complexion nature with metal ions and site-specific absorption (upper GIT). The plasma half-life of the drug is $\sim 1.5 \mathrm{~h}$. Absorption after an oral dose is relatively rapid (Tmax $\sim 1 \mathrm{hr}$ ) and occurs throughout the upper GIT. The site-specific absorption (upper GIT) of risedronate sodium provides a strong rationale for its use as a model drug for gastroretentive dosage form [7].

The aim of the study was to prepare as mucoadhesive microspheres to reduce dosing frequency and to effectively reduce variations in plasma drug concentration by delivering the drug in a controlled and reproducible fashion. 


\section{MATERIALS AND METHODS}

\section{Materials}

Risedronate sodium was obtained as a generous gift sample from JPN pharmaceutical Ltd. (Central Mumbai, India). Sodium alginate, noctanol, calcium chloride and span 80 were purchased from Research-lab fine Chem industries. All other chemicals and reagents used were of analytical reagent grade and were procured from commercial sources.

\section{Preparation of microspheres of risedronate sodium}

The emulsification crosslinking method is used to prepare microspheres of risedronate sodium using calcium chloride as a crosslinking agent. In brief, risedronate sodium was dispersed in an aqueous solution containing $3 \%, \mathrm{w} / \mathrm{v}$ sodium alginate. The solution was dispersed in $\mathrm{n}$ octanol containing $2 \%, \mathrm{v} / \mathrm{v}$ span 80 using a mechanical stirrer (Remi, $\mathrm{RQ127A/D)} \mathrm{at} \mathrm{1800rpm.} \mathrm{The} \mathrm{ratio} \mathrm{of} \mathrm{the} \mathrm{aqueous} \mathrm{to} \mathrm{n-octanol} \mathrm{phase}$ used was 1:20. The resultant w/o emulsion was stirred for $30 \mathrm{~min}$. This was followed by the dropwise addition of the $4 \%$, w/v calcium chloride solution under mechanical agitation of different speeds using homo dispensers for another $5 \mathrm{~min}$. The microspheres were collected by vacuum filtration, washed three times with isopropyl alcohol and dried in an air at room temperature [8].

The batches were prepared according to the formulation design (table 3).

\section{Optimization}

The optimization of pharmaceutical formulations with regard to one or more attribute has always been a subject of importance and attention for those engaged in formulation research. The objective is to produce a mathematical model that describes the responses. In general, the procedure consists of preparing a series of formulations, varying the concentrations of the formulation ingredients in some systematic manner (Bolton, 2004). The various computations for the current optimization study were performed using Design Expert ${ }^{\circledR}$ software (Design Expert trial version 7.0.0; State-Ease Inc., Minneapolis, MN, USA). A three-factor two-level full factorial design was used for the systemic study of combination of Polymers.

\section{$2^{3}$ full factorial design}

If three factors A, B, and C, each at two levels, are to be investigated, eight trials are necessary for a full factorial design, as shown in (table 3). This is also called a $2^{3}$ experiment, three factors, each at two levels. A $2^{3}$ full factorial design was constructed where the polymer, inlet temperature, aspirator rate was selected as factors. The levels of these factors were selected on the basis of initial studies and observations. All the other formulation aspects and processing variables were kept invariant throughout the study period. Polynomial models including interaction and quadratic terms were generated for the entire response variables using multiple linear regression analysis (MLRA) approach. The polynomial equations can be used to draw a conclusion after considering the magnitude coefficient and the mathematical sign that the coefficient carries. A high positive or negative value in the equation represents that by making a minor change in the setting of that factor one may obtain a significant change in the dependent variable.

The statistical validity of the polynomials was established on the basis of analysis of variance (ANOVA) provision in the Design Expert software. Level of significance was considered at $\mathrm{p}<0.05$. The bestfitting mathematical model was selected based on the comparison of several statistical parameters, including the coefficient of variation (CV), the multiple correlation coefficients (R2), the adjusted multiple correlation coefficient (adjusted R2), and the predicted residual sum of squares (PRESS), provided by the software. PRESS indicates how well the model fits the data, and for the chosen model, it should be small relative to the other models under consideration. The 3-D response surface graphs and the 2-D contour plots were also generated by the Design Expert $\AA$ software (8.0.4.). These plots are very useful to see interaction effects of the factors on responses.

\section{Data analysis of factorial design}

Once the experimental design is applied and the observations are made then the analysis of the data obtained is carried out by one of the two methods;

Polynomial Equation: It is the most common technique for quantitative factor analysis. The first step is to transform real values, i.e. to obtain coded values. The equation is carried out using transformed values and is given by

\section{$Y=\beta 0+\beta 1 X 1+\beta 2 X 2 \beta 3 X 3+\beta 12 X 1 X 2+\beta 13 X 1 X 3+\beta 23 X 2 X 3+\beta 23 X 1 X 2 X 3+\ldots$}

Where $\mathrm{Y}$ is the measured response, $\mathrm{Xi}$ is the level (e. g. Drug: polymer weight ratio, the concentration of calcium chloride, cross linking time) of the $\mathrm{i}^{\text {th }}$ factor, $\beta 0$ is the intercept $\beta 1, \beta 2, \beta 3, \beta 12,{ }_{2} 3$ and $\beta 123$ are regression coefficient for the variables and interaction between the variables.

Table 1: Investigating ranges of variables for risedronate sodium and sodium alginate microspheres

\begin{tabular}{lll}
\hline S. No. & Low level & High level \\
\hline $\mathrm{X}_{1}$ & $0.5: 1$ & $1: 1$ \\
$\mathrm{X}_{2}$ & 2 & 4 \\
$\mathrm{X}_{3}$ & 5 & 10 \\
\hline
\end{tabular}

Table 2: Layout of $2^{3}$ full factorial design

\begin{tabular}{|c|c|c|c|c|c|c|c|c|}
\hline Variable & F1 & F2 & F3 & F4 & F5 & F6 & F7 & F8 \\
\hline $\mathrm{X} 1$ & -1 & 1 & 1 & -1 & 1 & -1 & -1 & 1 \\
\hline $\mathrm{X} 2$ & 1 & 1 & 1 & -1 & -1 & -1 & 1 & -1 \\
\hline X3 & 1 & 1 & -1 & 1 & 1 & -1 & -1 & -1 \\
\hline
\end{tabular}

Table 3: Formulation for risedronate sodium microspheres

\begin{tabular}{llll}
\hline Formulation & $\mathbf{X 1}$ & $\mathbf{X 2}$ & $\mathbf{X 3}$ \\
\hline $\mathrm{F}_{1}$ & $0.5: 1$ & 4 & 10 \\
$\mathrm{~F}_{2}$ & $1: 1$ & 4 & 10 \\
$\mathrm{~F}_{3}$ & $1: 1$ & 4 & 5 \\
$\mathrm{~F}_{4}$ & $0.5: 1$ & 2 & 10 \\
$\mathrm{~F}_{5}$ & $1: 1$ & 2 & 10 \\
$\mathrm{~F}_{6}$ & $0.5: 1$ & 2 & 5 \\
$\mathrm{~F}_{7}$ & $0.5: 1$ & 4 & 5 \\
$\mathrm{~F}_{8}$ & $1: 1$ & 2 & 5 \\
\hline
\end{tabular}




\section{Formulation of Microspheres as per $\mathbf{2}^{\mathbf{3}}$ factorial design}

Independent variables: $\mathrm{X} 1$ = Drug: Polymer weight ratio (mg)

$\mathrm{X} 2=$ Concentration of $\mathrm{CaCl}_{2}(\%)$

$\mathrm{X} 3$ = Cross-linking time (min)

Dependent variables: $\mathrm{Y} 1$ = Particle size (micron)

$\mathrm{Y} 2$ = In vitro mucoadhesion (\%)

\section{Evaluation of prepared microspheres}

\section{Percentage yield}

The percent yield of microsphere was calculated based on the amount of drug and polymer used for the formulation of microspheres [9]. The percentage yield was calculated from Eq. 1

$$
\% \text { Yield }=\frac{\text { Total weight of microspheres }}{\text { Theoretical weight of drug and polymer }} \times 100
$$

\section{Entrapment efficiency}

$50 \mathrm{mg}$ equivalent of risedronate sodium containing microspheres was dissolved in $10 \mathrm{ml}$ of water. The absorbance was measured by a UV spectrophotometer (Agilent, carry 60UV-Vis) at $224 \mathrm{~nm}$ [10]. The $\%$ entrapment efficiency was calculated from Eq. 2

$$
\% \text { Entrapment Efficiency }=\frac{\text { practical amount of drug }}{\text { Theoretical amount }} \text { X 100-- }
$$

The results were analyzed in triplicate and standard deviations are reported.

\section{Particle size analysis}

Particle size analysis of risedronate sodium was determined by an optical microscope. A little amount of dry microspheres was dispersed in cyclohexane (5cc) (cyclohexane was selected due to insolubility of polymer and drug in it). Then the suspension was sonicated for 5s. A small drop of the suspension, thus obtained was placed on a clean glass slide. The slide containing sodium alginate microspheres was mounted on the stage of the microscope, and the diameter of molecules was evaluated utilizing a calibrated ocular micrometre. The particle size analysis was carried out using a microscopic image analysis technique [11].

The results were analyzed in triplicate and standard deviations are reported.

\section{Particle shape and surface morphology}

The morphology of the prepared microspheres was investigated by scanning electron microscopy (Diya labs, New Mumbai). The microspheres were fixed on adequate supports and coated with gold under an argon atmosphere using a gold sputter module in a highvacuum evaporator. Observations under different magnifications were performed at $15 \mathrm{kV}[9,10]$.

\section{In vitro drug release study}

The drug release rate from mucoadhesive microspheres was carried out using USP dissolution apparatus I (ED2L Electrolab). A weight of mucoadhesive microspheres corresponding to $58 \mathrm{mg}$ drug was filled into a capsule and placed in the basket. Dissolution media was $900 \mathrm{ml}$ of $0.1 \mathrm{~N} \mathrm{HCl}$ maintained at $37 \pm 0.5^{\circ} \mathrm{C}$ and stirred at $100 \mathrm{RPM} .5 \mathrm{ml}$ sample was withdrawn at suitable time intervals for $12 \mathrm{~h}$ and $5 \mathrm{ml}$ fresh dissolution medium was replaced after each withdrawal. These samples were analyzed for the drug spectrophotometrically at $224.5 \mathrm{~nm}$ [10].

\section{In vitro mucoadhesive strength}

A freshly cut $2 \mathrm{~cm}$ piece of goat stomach mucosa was obtained and cleaned by washing with an isotonic saline solution. One hundred milligrams of microspheres were placed on the mucosal surface was fixed over polyethene support. The microspheres were brought in contact with simulated electrolytes (SNES: an aqueous solution containing $8.77 \mathrm{mg} / \mathrm{ml} \mathrm{NaCl}, 2.98 \mathrm{mg} / \mathrm{ml} \mathrm{KCl}$ and $0.59 \mathrm{mg} / \mathrm{ml} \mathrm{CaCl} 2$ per liter). The goat stomach mucosa was washed with phosphate buffer ( $\mathrm{pH} \mathrm{6.6)}$ at the rate of $5 \mathrm{ml} / \mathrm{min}$ using a peristaltic pump. Sixty minutes after application of microspheres, the concentration of drug in collected perfusate was determined spectrophotometrically. The amount of microspheres corresponding to the drug amount in perfusate was calculated. The amount of adhered microspheres was estimated from the difference between the applied microspheres amount and the amount of flowed microspheres [12]. The percent mucoadhesion was calculated from Eq. 3

$$
\% \text { Mucoadhesion }=\frac{\text { Amount of drug in washout liquid }}{\text { Amount of drug in applied microsphere }} \times 100
$$

The results were analyzed in triplicate and standard deviations are reported.

\section{RESULTS AND DISCUSSION}

\section{Preparation of microspheres}

Eight batches were prepared by the emulsification crosslinking method as per factorial design, in which Drug: Polymer weight ratio (mg) (X1), Concentration of $\mathrm{CaCl}_{2}(\%)(\mathrm{X} 2)$, Cross-linking time (min) (X3), were selected as independent variables and (micron) particle size (Y1), \% In vitro mucoadhesion (Y2) of the microspheres were taken as the dependent variables were treated using design expert software.

\section{Percentage Yield}

The percentage yield of different batches was determined by weighing the microspheres after drying. The percentage yields of different formulations were found to be in the range of $61.29 \%$ to $89.33 \%$. This high yield of production is may be due to all the polymer is available for gelation into a cross linking agent.

\section{Entrapment efficiency}

The drug entrapment efficiency of different batches of microspheres was found in the range of $36.05 \%$ to $62.58 \%$. The maximum percent of drug entrapment efficiency was found to be in the formulation batch F3. The \% entrapment efficiency showed dependence on drug loading, the amount of cross-linking agent and time of cross-linking. The formulations loaded with the higher amount of drug exhibited higher entrapment efficiencies. The entrapment efficiency, however, showed an inverse relationship with the increase in cross link density, which will reduce the free volume spaces within the polymer matrix and hence, a reduction in entrapment efficiency.

\section{Particle size analysis}

The particle size of microspheres varied somewhat among the formulation due to variation in the composition of formulations. Formulation F6 showed a relatively large size, i.e. $54.32 \mu \mathrm{m}$ and formulation F1 showed relatively small size i.e. $32.85 \mu \mathrm{m}$. The (table 4) shows the mean particle size of various batches. Preliminary studies showed that as the concentration of polymer was increased, the particle size also proportionally increased. Low alginate concentrations $(1 \%, \mathrm{w} / \mathrm{v}$ and $2 \%, \mathrm{w} / \mathrm{v})$ resulted in clumping of microspheres, whereas high sodium alginate concentration $(4 \%$, $\mathrm{w} / \mathrm{v}$ ) resulted in the formation of discrete microspheres with size 90 $\mu \mathrm{m}$. This could be attributed to an increase in the relative viscosity at a higher concentration of polymer and the formation of larger particles during emulsification. Hence the optimized concentration of $3 \%, w / v$ was selected for preparing the different batches of the microspheres. The size of microspheres was increased with an increased with an increase drug loading. This can be attributed to the corresponding increase in viscosity of drug-polymer dispersion comprising the internal phase of the emulsion. A similar increase in the size of microspheres was also observed to increase in calcium chloride concentration as well as cross-linking time. The addition of a higher amount of $\mathrm{Ca}^{2+}$ will result in relatively more cross-linking of the gluronic acid units of sodium alginate, thereby leading to the formation of larger microspheres. Similarly, increasing the cross linking time will increase the extent of cross-linking and thereby increase the particle size.

\section{Particle shape and surface morphology}

Morphological analysis of the risedronate sodium microsphere was carried out by optical microscopy. The microspheres were found to be discrete and spherical in shape. 
Table 4: Trial batches evaluation

\begin{tabular}{llll}
\hline Batch No. & P. S. \pm SD* & P. I. M. \pm SD* & P. Y. \\
\hline $\mathrm{F}_{1}$ & $32.85 \pm 0.774$ & $82.24 \pm 0.72$ & 89.33 \\
$\mathrm{~F}_{2}$ & $39.12 \pm 1.447$ & $75.12 \pm 1.04$ & 61.29 \\
$\mathrm{~F}_{3}$ & $34.15 \pm 0.603$ & $78.13 \pm 0.83$ & 82.23 \\
$\mathrm{~F}_{4}$ & $47.94 \pm 2.12$ & $72.52 \pm 1.07$ & 80.33 \\
$\mathrm{~F}_{5}$ & $37.64 \pm 0.732$ & $75.12 \pm 0.64$ & 86.67 \\
$\mathrm{~F}_{6}$ & $54.32 \pm 0.612$ & $70.24 \pm 1.05$ & 66.12 \\
$\mathrm{~F}_{7}$ & $36.18 \pm 0.835$ & $76.75 \pm 0.77$ & 84.230 \\
$\mathrm{~F}_{8}$ & $46.45 \pm 0.961$ & $68.15 \pm 0.37$ & 79.620 \\
\hline
\end{tabular}

*SD standard deviation, n=3, P. S.: particle size, P. I. M.: percent in vitro mucoadhesion, P. Y.: percent yield, P. E. E.: percent entrapment efficiency

\section{In vitro drug release}

To determine the release risedronate sodium is in a controlled manner by formulating the polymeric microspheres, in vitro drug dissolution studies were carried out in $0.1 \mathrm{~N} \mathrm{HCl}$ solutions using USP dissolution apparatus I. F1 batch shows significant cumulative percent drug release from microspheres this may be due to smaller particle size, which leads to larger area of contact with the dissolution medium favour more rapid dissolution of the drug in comparison with larger particles. Drug release of all eight formulations is shown in fig. 1.

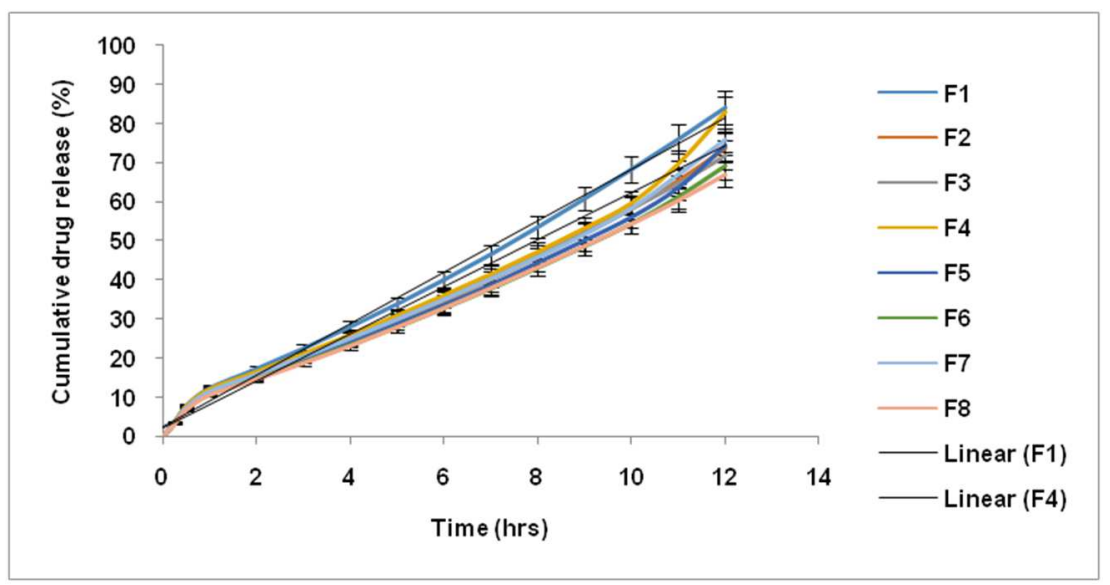

Fig. 1: Graphical representation of comparative release profile of eight formulations [mean $\pm S D, n=3$ ]

\section{In vitro mucoadhesion study}

Mucoadhesion studies were followed to ensure the adhesion of the formulation to the stomach mucosa for a prolonged period of time at the site of absorption. The \% In vitro mucoadhesion of all eight batches were found to be in the range of $68.150 .37 \%$ to $82.24+0.72 \%$. Selected batch F1 shows higher percent in vitro mucoadhesion this may be due to sodium alginate is practically insoluble in aqueous acidic solution; whereas, its solubility, hydration and bioadhesion properties were increased in the simulated intestinal fluid due to ionization of the carboxyl acid group and other functional groups present in the polymer. This increased solubility allowed more solvent to penetrate the polymeric coat, producing a viscous gel and increasing the mucoadhesion property.

\section{Fourier transform infrared spectroscopy (FTIR)}

To check the compatibility of the drug with a polymer, infrared spectra of drug, polymers and mixture of drug-polymer were studied by using FTIR-ATR (spectrum 2, Perkin Elmer). The IR spectrum is shown in fig. 2. Indicates that there is no physicochemical interaction in between the drug and polymer which suggest that the polymer was compatible with FTIR spectra of risedronate sodium.

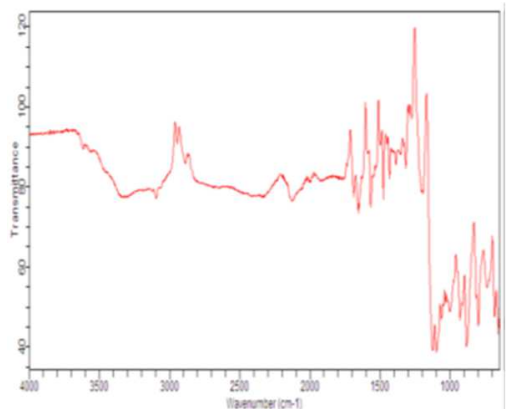

(A)

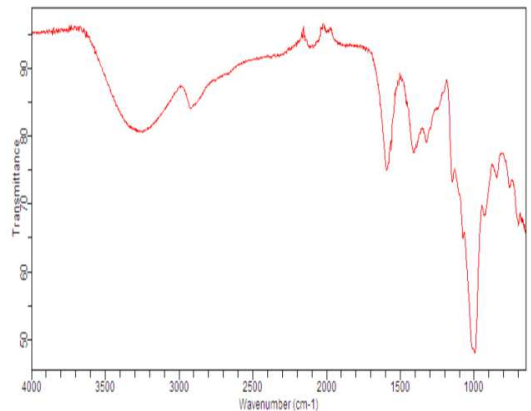

(B)

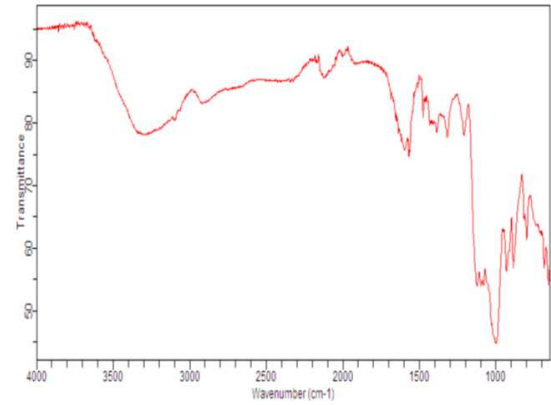

(C)

Fig. 2: Fourier transform infrared (FTIR) spectra of risedronate sodium (A), sodium alginate (B), risedronate sodium and polymer physical mixture (C) 


\section{Differential scanning calorimetric (DSC) study}

Differential scanning calorimetry (DSC) thermogram of risedronate sodium is shown in the fig. 3. Differential scanning calorimetric (DSC) studies indicate a sharp endothermic peak at $268.09^{\circ} \mathrm{C}$ corresponding to the melting point of the sample which matches with the melting point of risedronate sodium indicating the purity of the drug. From the differential scanning calorimetric (DSC) overlay thermogram of pure drug and physical mixture with sodium alginate; it can be concluded that the occupants and drug don't have any interaction with each other. Also the drug did not form a complex with the excipients as the endothermic peaks remained unchanged in position.

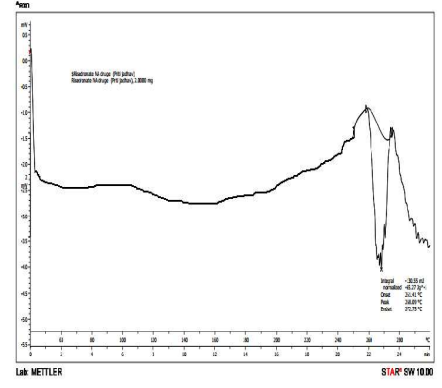

(A)

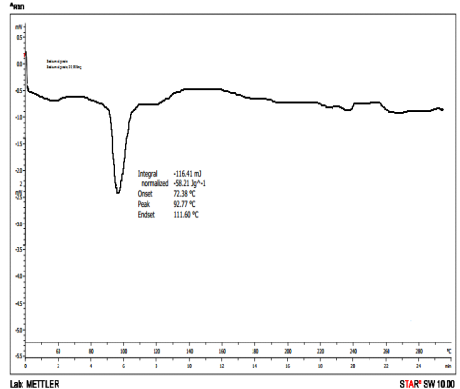

(B)

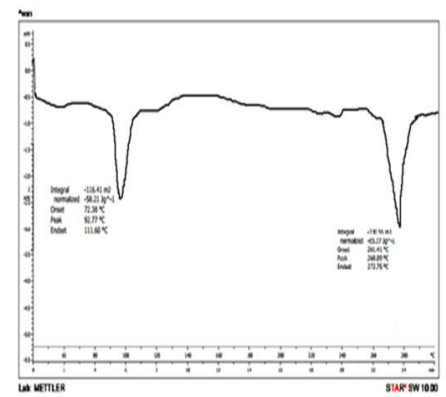

(C)

Fig. 3: Differential scanning calorimetry (DSC) curves of risedronate sodium (A), sodium alginate (B), curves of Microsphere formulation (C)

\section{Experimental design and data analysis}

The aim of the present work was to achieve optimized formulations for risedronate sodium microspheres by determining the effects of some important factors (variables) and their interactions during the process of preparation on microspheres physicochemical properties. Meanwhile,

\section{Particle size}

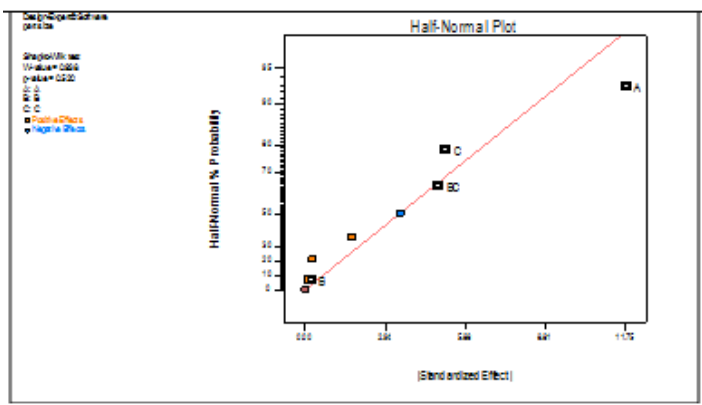

Half-Normal plot

\section{Analysis of variance and model equations}

In the next step, the significance of this influence was statistically confirmed by ANOVA Test. According to applied $2^{3}$ experimental design, including 8 experiments were performed to optimize the formulation method of microspheres to get maximum entrapment efficiency in terms of response. The obtained results were entered in design expert software 8.0.4. and a model equation was obtained to the microspheres were being processed; the impact of different factors had been evaluated by making changes in their quantity. Finally, three of the most significant factors had been chosen as the independent variables. According to a $2^{3}$ factorial design and considering these three variables, an experimental matrix was performed by using design expert 8.0.4 software in which 8 experiments were performed.

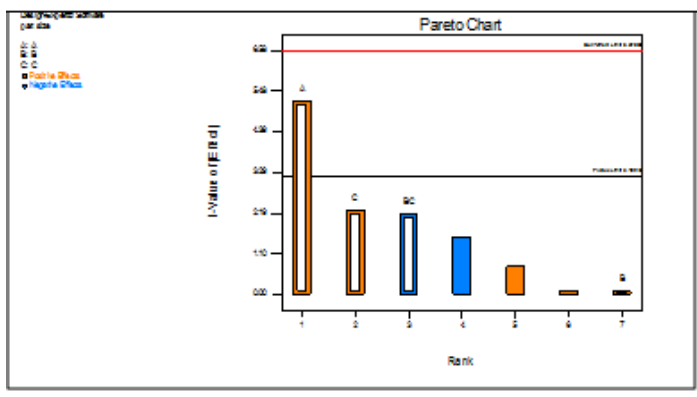

Pareto chart

get the fit result for particle size. The final model in terms of coded factors is,

Particle Size $=+41.08+5.88^{*} \mathrm{~A}+0.099^{*} \mathrm{~B}+2.57^{*} \mathrm{C}-2.43^{*} \mathrm{~B} * \mathrm{C}$

The model F-value of 9.30 implies the model is significant.

Values of "Prob>F" less than 0.0500 indicates model terms are significant. In this case, A are significant model terms.

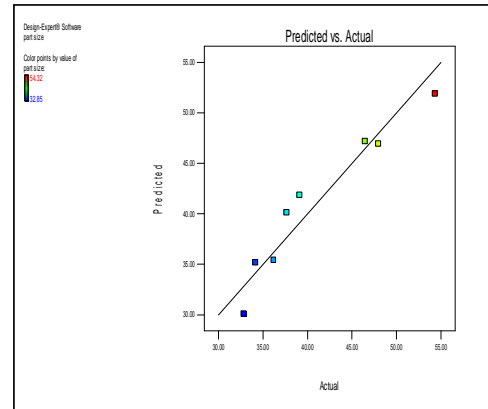

(A)

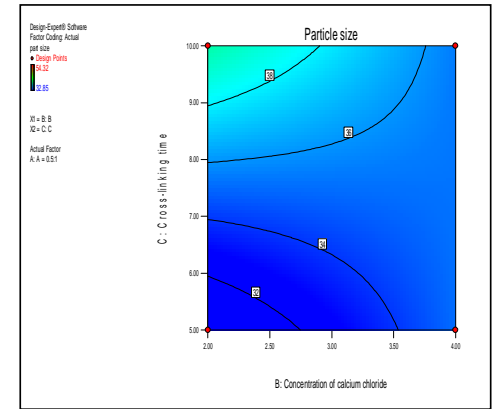

(B)

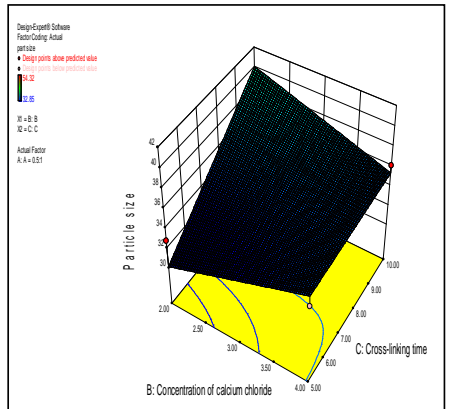

(C)

Fig. 4: (A): Predicted Vs. Actual values of particle size, (B): Contour plot shows the effect of concentration of calcium chloride (B) and cross-linking time (C) on the particle size, (C): 3D contour plot shows the effect of concentration of calcium chloride (B) and cross-linking time (C) on the particle size 
In vitro mucoadhesion

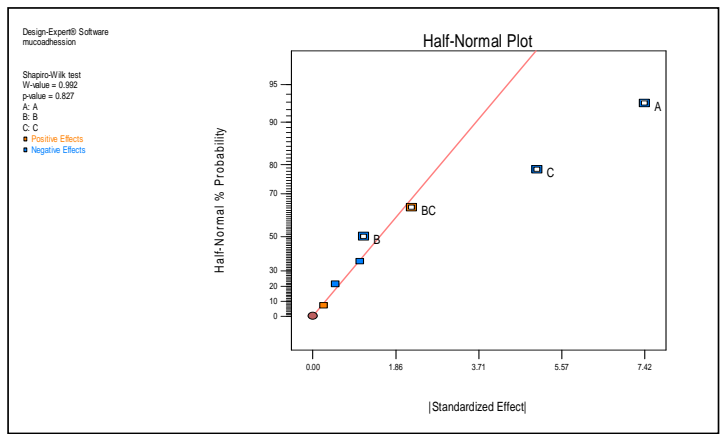

Half-normal plot

\section{Analysis of variance and model equations}

In the next step, the significance of this influence was statistically confirmed by ANOVA Test. According to applied $2^{3}$ experimental design, including 8 experiments were performed to optimize the formulation method of microspheres to get maximum in vitro mucoadhesion in terms of response. The obtained results were entered in design expert software 8.0.4. and a model equation was obtained to get the fit result for in vitro mucoadhesion. The final model in terms of coded factors is,

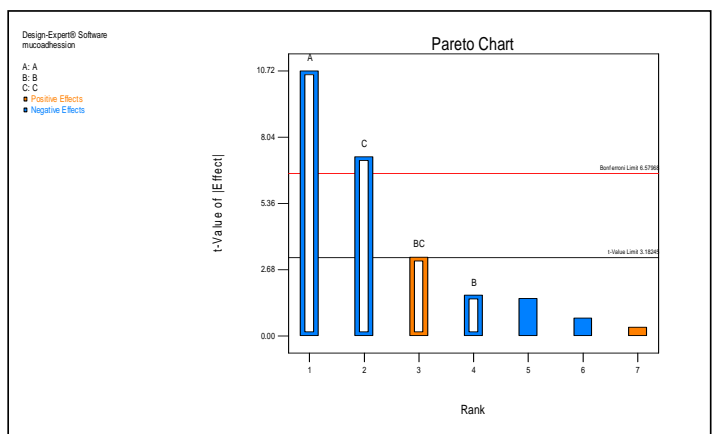

Pareto chart

In vitro mucoadhesion $=+74.49-3.71 * \mathrm{~A}-0.57^{*} \mathrm{~B}-2.51 * \mathrm{C}+1.11^{*} \mathrm{~B} * \mathrm{C}$

The model F-value of 45.13 implies the model is significant. Values of "Prob>F" less than 0.0500 indicates model terms are significant. In this case A, C and BC are significant model terms. Values greater than 0.1000 indicates the model terms are significant.

\section{Optimized formulations}

$F_{1}$ formulation was selected as optimized formulation on the basis of the optimum result of particle size and in vitro mucoadhesion.

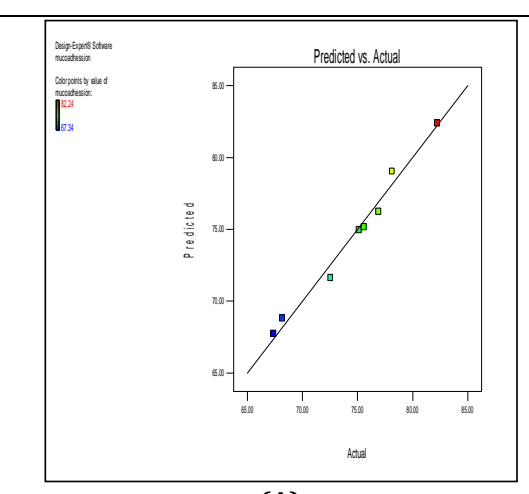

(A)

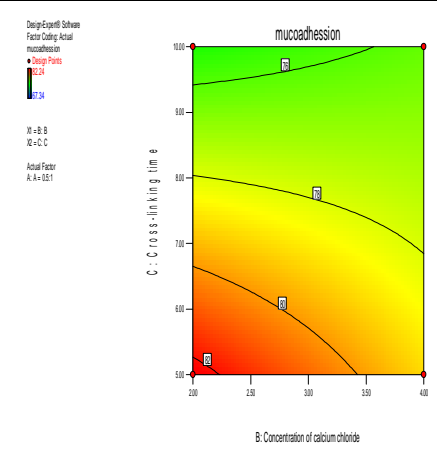

(B)

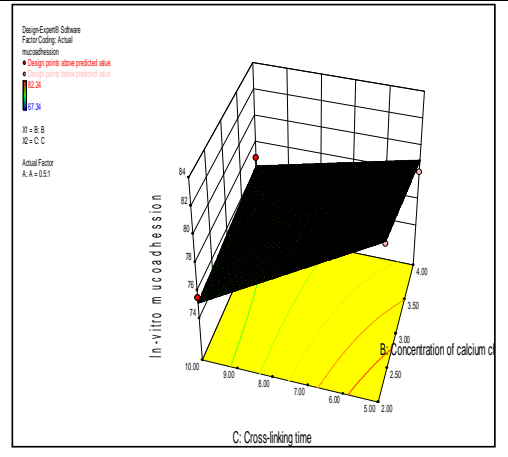

(C)

Fig. 5: (A): Predicted Vs. actual values of in vitro mucoadhesion, (B): Contour plot shows the effect of concentration of calcium chloride (B) and cross-linking time (C) on the in vitro mucoadhesion, (C): 3D Contour plot shows the effect of concentration of calcium chloride (B) and cross-linking time (C) on the in vitro mucoadhesion

Table 5: Predicted drug-loading and related obtained response from three of selected formulations

\begin{tabular}{lllllll}
\hline Solution No. & $\mathrm{X} 1$ & $\mathbf{X 2}$ & $\mathbf{X 3}$ & Y1 & \multicolumn{1}{c}{ Y2 } \\
\hline & & & & Predicted & Obtained & \multicolumn{1}{l}{ Predicted } \\
response & Obtained & response & response \\
$\mathrm{F}_{1}$ & & & & 32.85 & 30.11 & 82.24 \\
\hline
\end{tabular}

From the obtained data, formulations $\mathrm{F}_{1}$ is selected as optimized formula and formulated times to confirm the optimized formulation. The selected formulation $F_{1}$ had has been chosen for particle size determination, entrapment efficiency and morphological evaluation by scanning electron microscopy (SEM), x-ray diffraction (XRD) and in vitro mucoadhesion study.

Evaluation of optimized batch

Table 6: Evaluation of optimized batch

\begin{tabular}{llll}
\hline Solution & P. E. E. \pm SD* & P. S. \pm SD* & P. C. D. R. \pm SD* \\
\hline $\mathrm{F}_{1}$ & $58.56 \pm 0.230$ & $32.85 \pm 0.774$ & 84.07 \\
\hline
\end{tabular}

*SD standard deviation, n=3, P. E. E.: Percent entrapment efficiency, P. S.: Particle size ( $\mu$ m), P. C. D. R.: Percent cumulative drug release

SEM: Morphology of the optimized batch was F1 studied by SEM analysis. The fig. 6 shows images of the optimized batch. SEM was done in Diya labs, (Airoli, New Mumbai) by scanning electron microscope. Photograph of SEM for showing that the drug may be present in the bulk of the microspheres and not surface associated. 


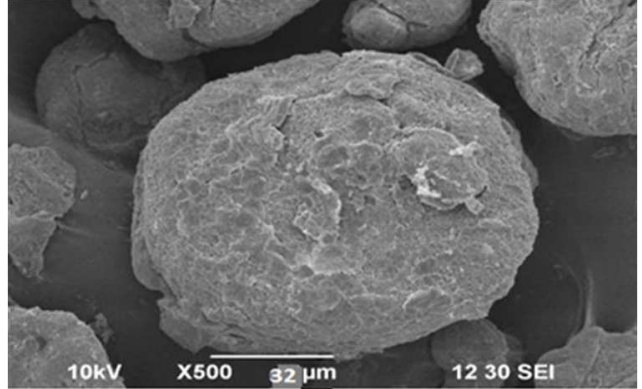

Fig. 6: Scanning electron microscopy (SEM) image of optimized batch

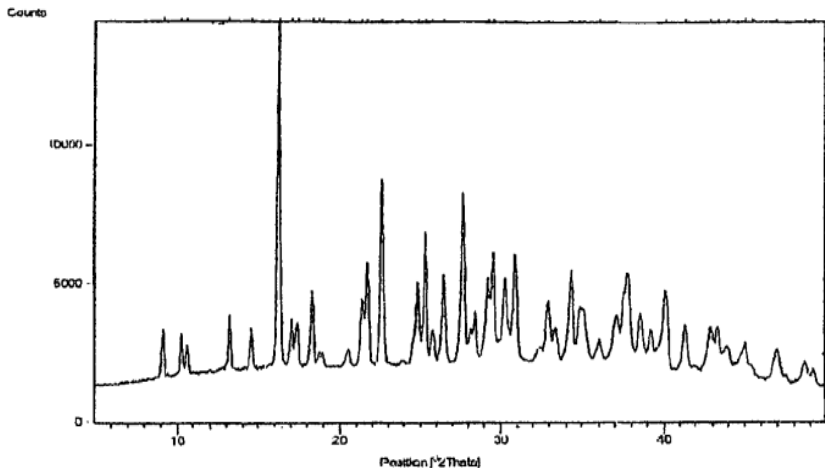

(A)

\section{X-ray diffraction (X-RD) studies}

$\mathrm{X}$-ray diffraction (XRD) analysis was carried by using x-ray diffraction (XRD) system. The x-ray diffraction (XRD) pattern for pure risedronate sodium and risedronate sodium loaded microspheres are shown in fig. 7, changes in the number of peaks and few diffuse peaks were observed in risedronate sodium loaded microspheres with reduced intensity to a greater extent as compared to x-ray diffraction (XRD) spectrum of pure drug, This indicates that drug particles are disperse at the molecular level in the polymer matrices so no indication of the crystalline nature of the drugs was observed in the drug loaded microspheres and it changes towards amorphous form. The increase in the amorphous nature of drug may be helpful in increasing solubility of pure drug leading to faster dissolution.

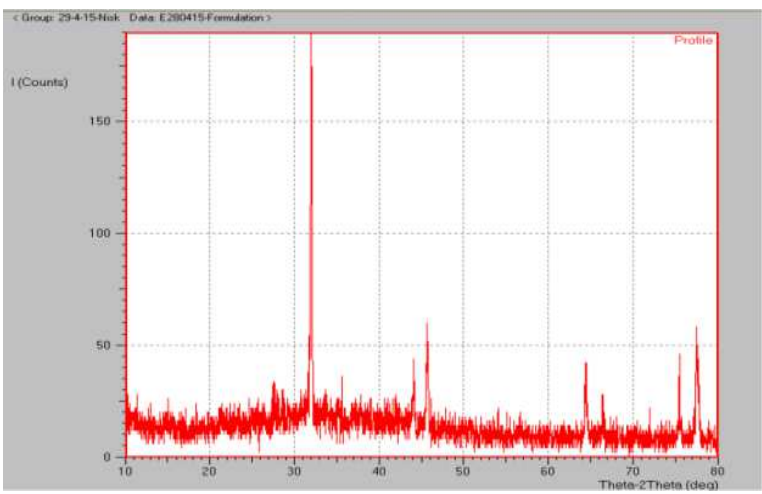

(B)

Fig. 7: X-ray diffraction (XRD) graph of risedronate sodium (A), x-ray diffraction (XRD) graph of microsphere formulations (B)

\section{Kinetic treatment to dissolution data}

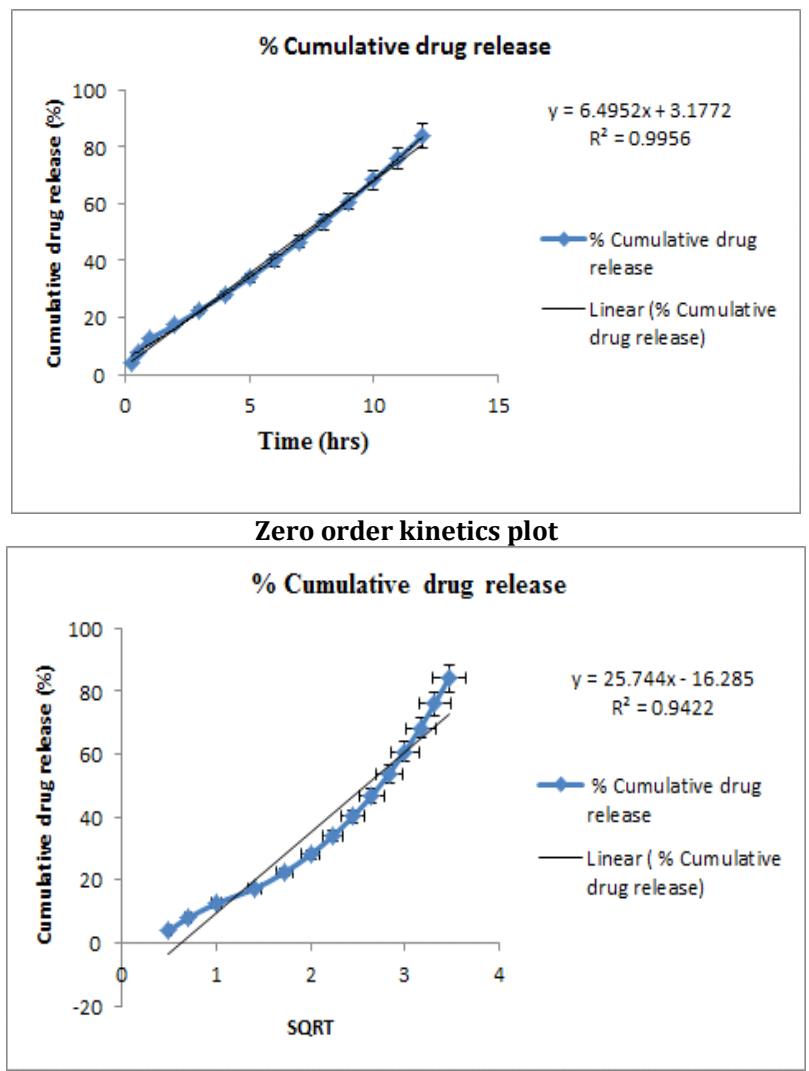

Higuchi model plot

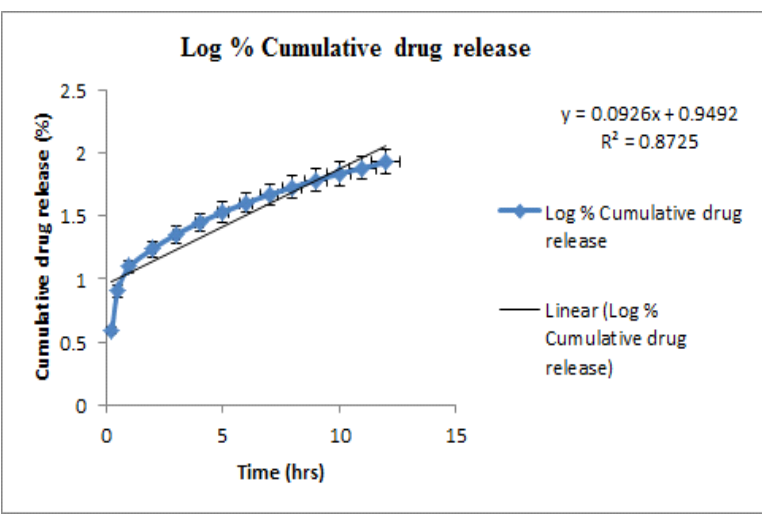

First order kinetics plot

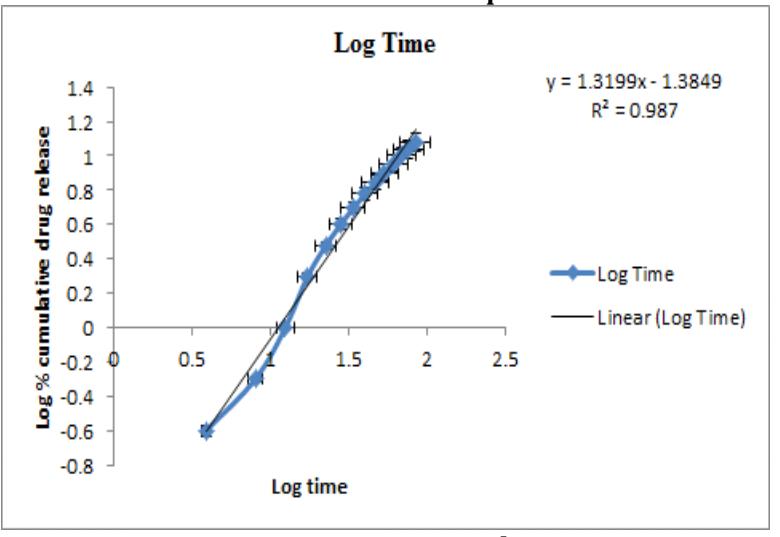

Korsmeyer peppas plot

Fig. 8: Kinetic treatment of dissolution data [mean $\pm S D, n=3]$ 


\section{Effect of temperature and humidity}

Effect of temperature and humidity of the prepared microspheres was carried out, by storing optimized formulation at $4 \pm 2{ }^{\circ} \mathrm{C}$, and at room temperature $45 \% \mathrm{RH}$ for $30 \mathrm{~d}$ in a stability chamber. Two parameters, namely residual entrapment efficiency and in vitro release studies were carried out. It is found that microspheres stored at room temperature are not stable, whereas stored at $4^{\circ} \mathrm{C}$ is stable given in (table 7). Microspheres at room temperature showed decreases in the residual entrapment efficiency and different release pattern.

Table 7: Effect of temperature and humidity on optimized batch

\begin{tabular}{|c|c|c|c|c|c|}
\hline Temperature condition & Parameters & Days & & & \\
\hline & & 0 & 7 & 14 & 28 \\
\hline $4 \pm 2{ }^{\circ} \mathrm{C}$ & Drug release (\%) in $14^{\text {th }}$ hrs & 71.24 & 76.59 & 88.45 & 93.45 \\
\hline
\end{tabular}

\section{ABBREVIATION}

GRDDS: Gastroretentive Drug Delivery System; EE: Entrapment Efficiency; HCL: Hydrochloric Acid; SEM: Scanning Electron Microscope; FTIR: Fourier Transform Infrared; UV: Ultraviolet; IR: Infrared; XRD: X-ray Diffraction; DSC: Differential Scanning Calorimetry

\section{CONCLUSION}

The mucoadhesive microspheres were formulated which aimed at an effective reduction in variation in plasma drug concentration by delivering the drug in a controlled and reproducible fashion of risedronate sodium, a potent pyridinyl bisphosphonate, used in the treatment of osteoporosis, and other bone disorders. Mucoadhesive microspheres are an attractive concept, in that the drug can be entrapped inside particles to be released at the mucosal surface, where the particles are sticking and enhances gastric residence time. SEM photographs showed that microspheres were almost spherical in shape with slight smooth surfaces. In vitro, mucoadhesive studies revealed that concentration of polymer in the microspheres, amount of crosslinking agent and time of crosslinking affect the in vitro mucoadhesion. It was concluded that as the concentration of polymer increased, the in vitro mucoadhesion was also increased. It was concluded that at the time of crosslinking and the volume of crosslinking agent increases, particle size decreases and the mucoadhesive strength increases.

\section{ACKNOWLEDGEMENT}

Authors are thankful to JPN Pharmaceutical Ltd (Central Mumbai, India) for providing a gift sample of risedronate sodium

\section{AUTHORS CONTRIBUTIONS}

All the author have contributed equally

\section{CONFLICTS OF INTERESTS}

There is no conflict of interest

\section{REFERENCES}

1. Mudie DM, Amidon GL, Amidon GE. Physiological parameters for oral delivery and in vitro testing. Mol Pharm 2010;7:1388-405.

2. Nayak AK, Malakar J, Sen KK. Gastroretentive drug delivery technologies: current approaches and future potential. J Pharm Edu Res 2010;1:1-12.

3. Mandal UK, Chatterjee B, Senjoti FG. Gastro-retentive drug delivery systems and their in-viva success: a recent update. Asian J Pharm Sci 2016;11:575-84.

4. Dasari A, Sellappan V. Formulation and evaluation of nevirapine mucoadhesive microspheres. Int J Pharm Pharm Sci 2015;7:342-8.

5. Sunitha S, Amareshwar P, Santhosh KM. A study on the effect of different cellulose polymers on release rate from tramadol loaded microspheres prepared by an emulsion solvent evaporation method. Asian J Pharm Clin Res 2010;3:35-9.

6. Beri C, Sood R, Gupta A. Review article stomach specific mucoadhesive microspheres as controlled drug delivery System. Int J Pharm Pharm Sci 2013;5:21-6.

7. Darna B, Jarathi AK, Gande S, Medipally V, Bomma R. Gastroretentive floating capsules of risedronate sodium: development, optimization, invitro and invivo evaluation in healthy human volunteers. Int J Pharm Sci Tech 2015;8:2835-42.

8. Wan LSC, Heng PWS, Chan LW. Drug encapsulation in alginate microspheres by emulsification. J Microencap 1992;9:309-16.

9. Shah V, Sharma M, Parmar V, Upadhyay U. Formulation of sildenafil citrate loaded nasal microspheres: an in vitro, ex vivo characterization. Int J Drug Delivery 2010;2:213-20.

10. Koland M, Jacob A, Prabhu P, Shetty NG. Mucoadhesive microspheres of famotidine for gastro retentive drug delivery. Int J Drug Dev Res 2012;4:59-64.

11. More HN, Hajare AA. Practical physical pharmacy. Career publications Nashik; 2010. p. 121.

12. Jain SK, Jain NK, Jain A, Jain D, Chaurasia M. Mucoadhesive chitosan microspheres for non invasive and improved nasal delivery of insulin. Indian J Pharm Sci 2007;69:498-504. 